\title{
Nanoscale
}

\section{Impact of oxygen exchange reaction at the ohmic interface in $\mathrm{Ta}_{2} \mathrm{O}_{5}$-based ReRAM devices}

Received 00th January 20xx Accepted 00th January 20xx

DOI: $10.1039 / x 0 x \times 00000 x$

www.rsc.org/

\author{
Wonjoo Kim ${ }^{a}$, Stephan Menzel ${ }^{a}$, Dirk J. Wouters ${ }^{b}$, Bernd Roesgen ${ }^{a}$, John Robertson ${ }^{c}$, Rainer \\ Waser $^{\mathrm{a}, \mathrm{b}}$ and Vikas Rana ${ }^{\mathrm{a}}$
}

Interface reactions constitute essential aspects of the switching mechanism in redox-based resistive random access memory (ReRAM). For example, the modulation of the electronic barrier height at the Schottky interface is considered to be responsible for the toggling of the resistance states. On the other hand, the role of the ohmic interface in the resistive switching behavior is still ambigious. In this paper, the impact of different ohmic metal-electrode (M) materials, namely W, $\mathrm{Ta}, \mathrm{Ti}$, and $\mathrm{Hf}$ on the characteristics of $\mathrm{Ta}_{2} \mathrm{O}_{5}$ ReRAM is investigated. These materials are chosen with respect to their free energy for metal oxide formation and, associated, their impact on the formation energy of oxygen vacancy defects at the $\mathrm{M} / \mathrm{Ta}_{2} \mathrm{O}_{5}$ interface. The resistive switching devices with $\mathrm{Ti}$ and $\mathrm{Hf}$ electrodes that have a negative defect formation energy, show an early RESET failure during the switching cycles. This failure process with $\mathrm{Ti}$ and $\mathrm{Hf}$ electrode is attributed to the accumulation of oxygen vacancies in the $\mathrm{Ta}_{2} \mathrm{O}_{5}$ layer, which leads to permanent breakdown of the metal-oxide to a low resistive state. In contrast, the defect formation energy in the $\mathrm{Ta}_{2} \mathrm{O}_{5}$ with respect to $\mathrm{Ta}$ and $\mathrm{W}$ electrodes is positve and for those highly stable resistive switching behavior is observed. During the quasi-static and transient-pulse characterization, the ReRAM devices with the W electrode consistently show a increased high resistance state (HRS) than with the Ta electrode for all RESET stop voltages. This effect is attributed to the faster oxygen exchange reaction at the W-electrode interface during the RESET process in accordance to lower stability of $\mathrm{WO}_{3}$ than $\mathrm{Ta}_{2} \mathrm{O}_{5}$. Based on these findings, an advanced resistive switching model, wherein also the oxygen exchange reaction at the ohmic M-electrode interface plays a vital role in determining of the resistance states, is presented.

\section{Introduction}

Redox-based resistive random access memory (ReRAM) is considered as a strong candidate to compete against conventional non-volatile memories such as NAND flash memory for mass storage application because of its lower power consumption, fast switching speed and down-scaling under $20 \mathrm{~nm}$ [1-3]. Metal-oxide based bipolar ReRAM devices consist of an insulating switching layer stacked between two asymmetric metal electrodes: one low work function (WF) ohmic electrode (OE) (which is also easily oxidizable), such as W, Ti, Hf, or Ta, and a high WF (inert) metal electrode, such as $\mathrm{Pt}$. The resistive switching mechanism relies on the motion of mobile ionic defects under the applied electrical field. While motion of metal cations has been evidenced and their role cannot be

\footnotetext{
a. Peter Grünberg Institute, Forschungszentrum Jülich $\mathrm{GmbH}, 52425$ Jülich, Germany and also with JARA-Fundamentals in Future Information Technology (E-mail: w.kim@fz-juelich.de).

b. Institute of Materials in Electrical Engineering and Information Technology II, RWTH Aachen University, 52074 Aachen, Germany.

c. Electronic Devices \& Materials (EDM) Group, Department of Electrical

Engineering, University of Cambridge, United Kingdom

tElectronic Supplementary Information (ESI) available: [details of any supplementary information available should be included here]. See
} DOI: 10.1039/x0xx00000x excluded [4], the most widely accepted model is based on (double) positively charged oxygen vacancy defects $V_{0}$. These $V_{O}$ 's are introduced in the switching layer during the initial forming step, which results in the formation of a filamentary region with a high concentration of oxygen vacancies between these two electrodes. The main process of $\mathrm{V}_{\mathrm{O}}$ defect generation during forming is thought to be extrinsic defect generation due to oxygen excorporation (Equation 1, following the Kröger-Vink notation [5]):

$$
\mathrm{O}_{\circ}{ }^{\mathrm{x}} \leftrightarrow \mathrm{Vö}+2 \mathrm{e}^{\prime}+1 / 2 \mathrm{O}_{2} \text { (g) }
$$

When the electrons are localized at the metal cations, (1) becomes:

$$
\mathrm{O}_{\mathrm{O}}{ }^{\mathrm{x}}+2 \mathrm{M}_{\mathrm{M}}^{\mathrm{x}} \leftrightarrow \mathrm{V}_{0}+2 \mathrm{M}_{\mathrm{M}}^{\prime}+1 / 2 \mathrm{O}_{2}(\mathrm{~g})
$$

The presence of the $V_{0}$, by a local redox reaction, changes the valence of the neighbouring metal cations in the metal oxide film (Note that the Kröger-Vink notation only lists relative charges, with the metal here changing from neutral to negative. In absolute charge notation, the cation may go e.g. from charge state $5+$ to $4+$ ), hence, it is termed as valence change memory or VCM.

Besides the forming process (Eq. 1), the $\mathrm{V}_{\mathrm{O}}$ 's are also introduced during processing by oxygen exchange between the oxide layer and the OE layer, i.e. oxygen is extracted from the metal oxide (MO) and 
oxidizes the $\mathrm{OE}$ metal $\left(=\mathrm{M}^{*}\right)$ to the formation of $\mathrm{M}^{*}$-oxide, see Equation 3 (for notation, $\mathrm{M}_{\mathrm{M}(\mathrm{MO})}$ ) stands for an $\mathrm{M}$ atom on a $\mathrm{M}$-metal site in the $\mathrm{MO}$ metal-oxide, while $\mathrm{M}^{*} \mathrm{M}^{*}\left(\mathrm{M}^{*}\right)$ stands for an $\mathrm{M}^{*}$ atom on a $M^{*}$-metal site in the $M^{*}$ metal etc.). Here, it is assumed that the $M^{*}$ oxidizes to valence +4 , i.e. forms a new phase $M^{*} O_{2}$ :

$$
\begin{aligned}
& 2 \mathrm{O}_{\mathrm{O}(\mathrm{MO})^{\mathrm{x}}}+4 \mathrm{M}_{\mathrm{M}(\mathrm{MO})^{\mathrm{x}}}+\mathrm{M}^{*} \mathrm{M}^{*}\left(\mathrm{M}^{*}\right)^{\mathrm{x}} \leftrightarrow \\
& 2 \mathrm{~V}_{\mathrm{O}(\mathrm{MO})^{\prime}}+4 \mathrm{M}_{\mathrm{M}(\mathrm{MO})^{\prime}}+\mathrm{M}^{*} \mathrm{O}_{2}+\mathrm{V}_{\mathrm{M}^{*}\left(\mathrm{M}^{*}\right)^{\prime \prime \prime \prime}+4 \mathrm{~h}^{\prime}}
\end{aligned}
$$

In case, the cap layer is already partly oxidized at the interface (to $\mathrm{M}^{*} \mathrm{O}_{2-\delta}$ ), as e.g. during device operation, the oxygen exchange corresponds to a reverse $V_{O}$ exchange, see equation (4):

$$
\begin{aligned}
& \mathrm{O}_{\mathrm{O}(\mathrm{MO})^{\mathrm{x}}}+2 \mathrm{M}_{\mathrm{M}(\mathrm{MO})^{\mathrm{x}}}+\mathrm{V}_{\mathrm{O}\left(\mathrm{M}^{*} \mathrm{O}\right)}{ }+2 \mathrm{M}^{*} \mathrm{M}^{*}\left(\mathrm{M}^{*} \mathrm{O}\right)^{\prime} \leftrightarrow \\
& \mathrm{V}_{\mathrm{O}(\mathrm{MO})}{ }{ }^{\prime}+2 \mathrm{M}_{\mathrm{M}(\mathrm{MO})^{\prime}}+2 \mathrm{M}^{*} \mathrm{M}^{*}\left(\mathrm{M}^{*} \mathrm{O}\right)^{\mathrm{x}}+\mathrm{O}_{\mathrm{O}\left(\mathrm{M}^{*} \mathrm{O}\right)^{\mathrm{x}}}
\end{aligned}
$$

The oxygen exchange with the $\mathrm{OE}$ during deposition introduces a high concentration of the $V_{O}$ located in the oxide film near the $O E$ electrode (so called defect "reservoir"). The resultant introduction of $V_{O}$ in the oxide film lowers the initial resistance and the forming voltage of the device. For a $\mathrm{Pt} / \mathrm{SrTiO}_{3} / \mathrm{Ti}$ VCM cell, it is demonstrated that the forming voltage depends on $\mathrm{SrTiO}_{3} / \mathrm{Ti}$ layer thickness ratio [7]. The $\mathrm{SrTiO}_{3}$ layer is further chemically reduced by a thicker $\mathrm{Ti}$ layer, which finally leads to an electroforming-free device. Most switching models, however, presume that during the RESET/SET switching process, only a redistribution of the $V_{O}$ within the oxide layer takes place keeping the total amount of the $V_{0}$ 's constant, i.e. no further oxygen exchange with the $\mathrm{OE}$ takes place during the switching process.

As mobile donors, the Vö's can influence the overall resistance of the ReRAM cell in two ways. First, the local conductivity of the metaloxide film increases with increasing $\mathrm{V}_{\mathrm{O}}$ concentration $\left[\mathrm{V}_{\mathrm{O}}\right]$ [8-10]. Second, the electrostatic barrier heights at the high WF metal/oxide interface are modulated by the local $V_{O}$ concentration due to the Schottky effect [11-13]. During the RESET/SET switching (applying positive/negative bias to the inert electrode), the $\mathrm{V}_{0}$ 's move away/towards the interface with the high WF electrode (in accordance with disrupting/restoring the filament). It is assumed that the OE forms an Ohmic contact with the metal-oxide layer, whereas in absence of a high concentration of Vö (disrupted filament) the high WF metal forms high Schottky barrier that dominates the overall resistance in the high resistive state (HRS). During the SET operation, the $V_{O}$ 's move to the high WF, where the increased $V_{0}$ concentration lowers the Schottky barrier and the device switches to the low resistive state (LRS). Hence, interfacial conduction strongly affects the resistive values in the different states of the oxide ReRAM $[14,15]$.

In this study, we analyzed $\mathrm{Ta}_{2} \mathrm{O}_{5}$-based ReRAM devices using different $\mathrm{OE}$ materials ( $\mathrm{W}, \mathrm{Ta}, \mathrm{Ti}$ and $\mathrm{Hf}$ ). Among various oxide switching materials, the $\mathrm{Ta}_{2} \mathrm{O}_{5}$-based ReRAMs exhibit excellent performance in switching speed [16], endurance[1, 17], retention [18] and low power [19-21]. The OE materials are chosen for the defect formation energy of oxygen vacancy $\left(E_{\mathrm{vo}}\right)$ with respect to the $\mathrm{Ta}_{2} \mathrm{O}_{5}$ layer. For the $\mathrm{Hf}$ and the $\mathrm{Ti}$, this $E_{\mathrm{VO}}$ is negative, whereas it is positive for the Ta and the $\mathrm{W}$. In this experiment, we have observed that the OE not only affects the forming voltage but also the RESET process. The devices with the $\mathrm{Hf}$ and the Ti electrode show an early RESET failure, whereas the devices with the Ta and the W OE show highly reliable switching behaviour. The $\mathrm{Pt} / \mathrm{Ta}_{2} \mathrm{O}_{5} / \mathrm{W}$ ReRAM devices show faster RESET process than the $\mathrm{Pt} / \mathrm{Ta}_{2} \mathrm{O}_{5} / \mathrm{Ta}$, which leads to higher $R_{\mathrm{HRS}} / R_{\mathrm{LRS}}$ ratio under the same bias conditions. These observations indicate that oxygen interchange with the OE plays an important role during the resistive switching process. Based on these findings, an advanced resistive switching model, where the oxygen exchange reaction at the OE interface plays a vital role in determining of the resistance states, is presented.

\section{Experimental}

In this study, $5 \mathrm{~nm}$-thick Titanium (Ti) and $30 \mathrm{~nm}$-thick Platinum (Pt) layers are deposited by sputtering on a thermally grown $450 \mathrm{~nm}$-thick $\mathrm{SiO}_{2}$ layer on Si substrate. Next, photolithography and dry-etching processes are used to pattern the Pt layer as bottom electrode. After the patterning, $7 \mathrm{~nm}$-thick $\mathrm{Ta}_{2} \mathrm{O}_{5}$ is deposited by reactive sputtering under a process gas mixture of argon (77\%) and oxygen (23\%) with an RF power of $116 \mathrm{~W}$ at a chamber pressure of $2.3 \times 10^{-2}$ mbar. Without breaking the vacuum, a $13 \mathrm{~nm}$-thick $\mathrm{OE}$ and a $25 \mathrm{~nm}$-thick Pt layer are deposited by RF and DC sputtering, respectively. In this experiment, four different OE layers, i.e. $\mathrm{W}, \mathrm{Ta}, \mathrm{Ti}$ and $\mathrm{Hf}$ are used. All deposition processes are performed at room temperature. To pattern the top electrode, photolithography with positive photo-resist is applied. After the photo-resist (a)

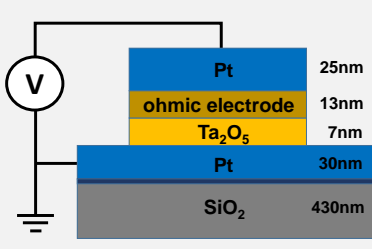

(c)

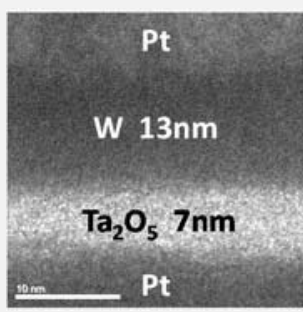

(b)
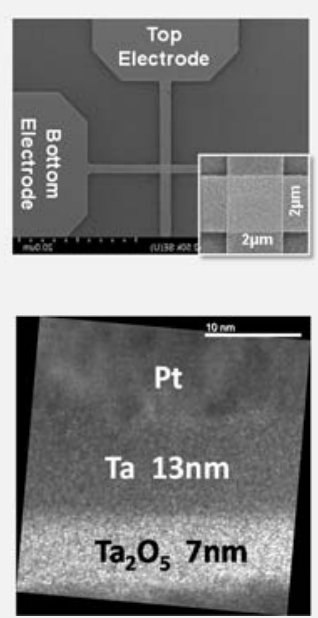

Fig. 1. (a) A schematic cross-sectional diagram with experimental split conditions with different ohmic electrode materials ( $\mathrm{W}, \mathrm{Ta}, \mathrm{Ti}$ and $\mathrm{Hf}$ ). (b) Scanning electron microscope image of the $2 \times 2 \mu \mathrm{m}^{2}$ ReRAM device in passive crossbar configuration. (c) TEM images of $\mathrm{W}$-electrode and Ta-electrode devices confirming target thickness of the corresponding layers. 
development, the top electrode is etched down with Reactive Ion Beam Etching (RIBE). This step leads to the $\mathrm{Pt} / \mathrm{Ta}_{2} \mathrm{O}_{5} / \mathrm{OE} / \mathrm{Pt}$ ReRAM device sketched in Fig. 1(a). A SEM image of the patterned passive crossbar structure with $2 \times 2 \mu \mathrm{m}^{2}$ device junction is shown in Fig. 1(b). The cross-sectional TEM images of the ReRAM devices with the Ta-OE and the W-OE layer confirm the thicknesses of corresponding device stacks in Fig. 1(c). The device characterization is performed using the Keithley 4200SCS under atmospheric conditions at room temperature.

\section{$V_{0}$ defect formation energies of used stacks}

The driving force of the interfacial chemical reaction (see eq. 3 ) is related to the energy of oxygen vacancy defect formation $\left(E_{\mathrm{vo}}\right)$ in the metal oxide at the OE interface [22-24]. Recently, Guo et al. calculated $E_{\mathrm{Vo}}$ in different metal oxides, and for different metal cap layers $[25,26]$. In these defect energy calculations, they made a difference between O-rich and O-poor conditions. The O-rich conditions actually apply to the metal oxide exposed to oxygen ambient (with atmospheric $\mathrm{pO}_{2}$ ). The O-poor condition corresponds to the metal-oxide in contact with an OE layer of the same metal as the oxide host metal $\left(M^{*}=M\right)$. In that case, the energy required for removing the $O$ from the metal oxide is nearly completely compensated by the (negative) free energy of oxidation of the metal cap, and the resultant defect formation energy is approximately 0. Fig. 2(a) shows the calculated $E_{\mathrm{vo}}$ values in $\mathrm{HfO}_{2}$ and $\mathrm{Ta}_{2} \mathrm{O}_{5}$ in contact with different $\mathrm{OE}$ (based on Fig. 5 of [27], where the energy values for the $\mathrm{Hf}$ and the $\mathrm{Ti}$ with respect to the $\mathrm{Ta}_{2} \mathrm{O}_{5}$ have been extrapolated based on available data in the reference $[25,26]$. Based on these calculations, the $E_{\mathrm{vo}}$ for the $\mathrm{Ta}_{2} \mathrm{O}_{5}$ layer is estimated to be $1.4 \mathrm{eV}$ for $\mathrm{W}$-electrode, $0.1 \mathrm{eV}$ for Ta-electrode. $-0.6 \mathrm{eV}$ for $\mathrm{Ti}$ electrode and $-1.5 \mathrm{eV}$ for $\mathrm{Hf}$-electrode. In general, as depicted in Fig. 2(a), the $E_{\mathrm{vo}}$ increases with the stability (i.e. lower free energy of oxide formation) of the host oxide (cf. parallel shift of defect energy for $\mathrm{Ta}_{2} \mathrm{O}_{5}$ and $\mathrm{HfO}_{2}$ ), and decreases according to the stability of the metal cap oxide (horizontal shift according to OE metal used. The $O$ chemical potential corresponds to the equilibrium oxygen partial pressure of the $M^{*} / M^{*} O$ couple, reflecting the relative $M^{*} O$ stability). It can be observed that a cap metal that has a more stable oxide than the oxide host metal (e.g., $\mathrm{Ta}_{2} \mathrm{O}_{5}$ capped with $\mathrm{Hf}$ and $\mathrm{Ti}$ ) results in a negative $E_{\mathrm{V}}$. A positive $E_{\mathrm{Vo}}$ is required for stable switching [27], as the negative $E_{\mathrm{Vo}}$ would result in a continuous increase of the $V_{O}$ in the switching film, resulting in a non-switchable, conducting film.

\section{Forming and switching properties}

The influence of the OE on the forming voltage was investigated by measuring 50 devices for each device stack. The forming process was performed by a positive DC voltage sweep (+3 V) applied to the OE with a current compliance $\left(I_{\mathrm{cc}}\right)$ of $1.0 \mathrm{~mA}$. The forming statistics of the four different device stacks are given in Fig. 2(b). A clear dependence of the forming voltage on the ohmic electrode material is observed. The lowest forming voltage is observed for the $\mathrm{Hf}$ electrode devices ( $V_{\text {Form }}=0.84 \mathrm{~V}$ in median), followed by the Ti-
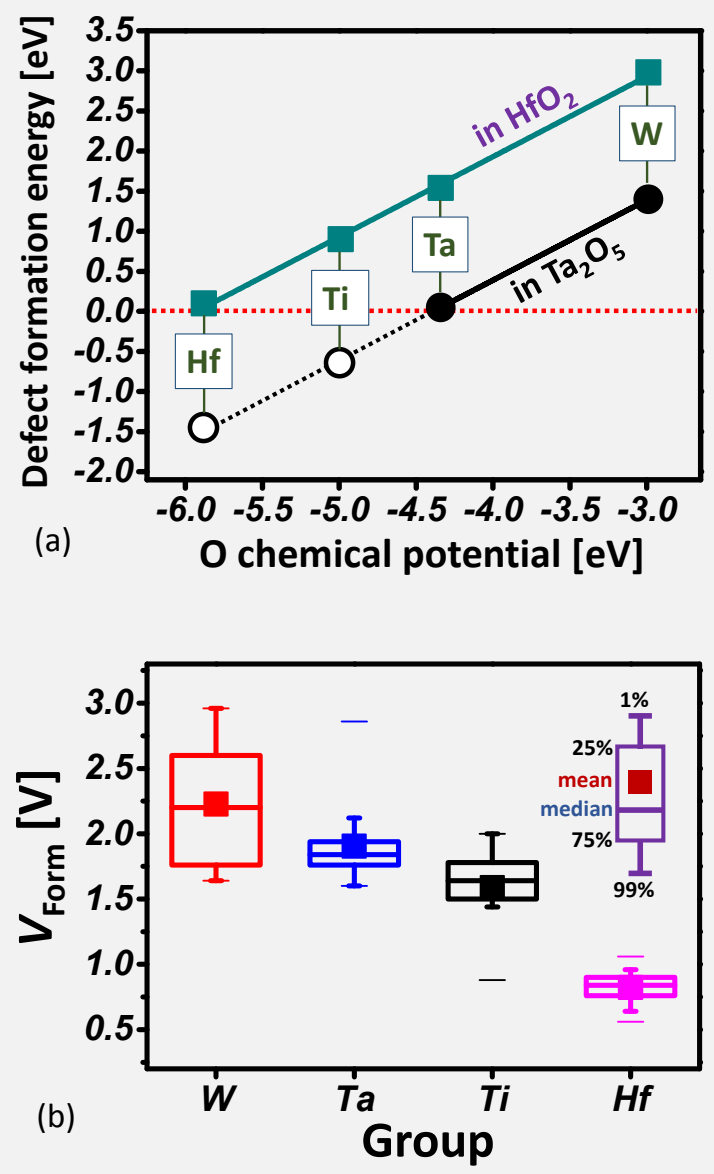

Fig. 2. (a) Interfacial defect formation energy for oxygen vacancy defects $\mathrm{E}_{\mathrm{vo}}$ in $\mathrm{Ta}_{2} \mathrm{O}_{5}$ and $\mathrm{HfO}_{2}$ as function of the oxygen chemical potential. This chemical potential is determined by the ohmic electrode present as indicated here for Ta, W, Ti and $\mathrm{Hf}$. (b) Forming voltage of the $\mathrm{Ta}_{2} \mathrm{O}_{5}$ ReRAM device for each ohmic metal electrode (W, $\mathrm{Ta}, \mathrm{Ti}$ and $\mathrm{Hf}$ )

electrode devices ( $V_{\text {form }}=1.64 \mathrm{~V}$ in median). The $\mathrm{W}$-electrode devices show the highest forming voltage $\left(V_{\text {Form }}=2.21 \mathrm{~V}\right.$ in median) followed by the Ta-electrode ones ( $V_{\text {Form }}=1.84 \mathrm{~V}$ in median). These forming voltage trends as shown in Fig. 2(b) clearly correspond with the oxygen vacancy defect formation energies at the respective $\mathrm{OE} / \mathrm{Ta}_{2} \mathrm{O}_{5}$ interfaces as shown in Fig. 2(a). During the switching, the ReRAM devices with the $\mathrm{Ti}$ - and the $\mathrm{Hf}-\mathrm{OE}$ show a failure in the RESET process within the first 20 cycles. This early RESET failure is shown in Fig. 3(a). The gray lines show the switching behavior of the $\mathrm{Ta}_{2} \mathrm{O}_{5}$ ReRAM with the Ti- and the Hf-OE before failure. After the RESET failure the ReRAM devices exhibit ohmic $I-V$ behavior and are stuck in the LRS regime. In contrast, stable switching operation has been observed with the $\mathrm{W}$ - and the Ta$\mathrm{OE}$ under the same biasing conditions as shown in Fig. 3(b). Average switching curves are shown in red for the W-OE and in blue for the Ta-OE devices. The occurrence of RESET failure or stable switching clearly correlates with (the sign of) $E_{\mathrm{V} o}$ at the $\mathrm{OE} / \mathrm{Ta}_{2} \mathrm{O}_{5}$ interface, suggesting that oxygen exchange takes place at this $\mathrm{OE} / \mathrm{Ta}_{2} \mathrm{O}_{5}$ interfaces during switching. This will be discussed with more details in the latter part of this study. 


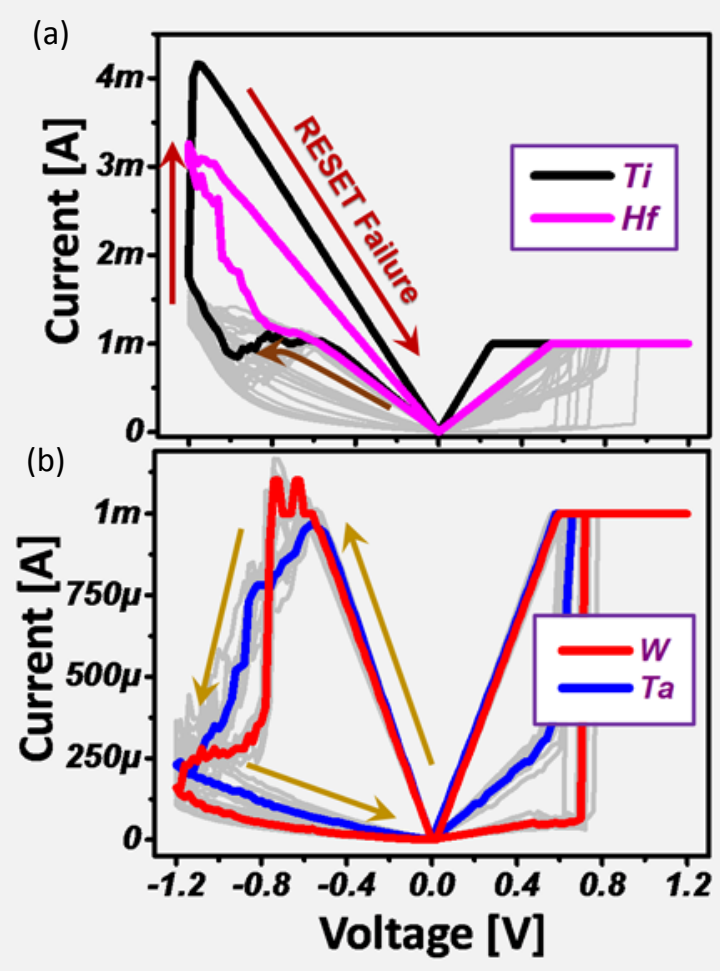

Fig. 3. At the given $I_{C C}$ of $1.0 \mathrm{~mA}$, the switching curves of $\mathrm{Ta}_{2} \mathrm{O}_{5}$ ReRAM with different $\mathrm{OE}$ and the maximum RESET voltage of $-1.2 \mathrm{~V}$ are examined. (a) Early RESET failures from "Hf" and "Ti" electrodes, (b) stable switching cycles of SET and RESET from "Ta" and "W" electrodes.

Since the Hf- and Ti-based devices show the early RESET failure, only the $\mathrm{W}$ - and Ta-OE based $\mathrm{Ta}_{2} \mathrm{O}_{5}$ ReRAM devices are further investigated and compared under identical bias conditions. Fig. 4(a) shows a typical $I-V$ characteristics of $\mathrm{Ta}_{2} \mathrm{O}_{5}$ device at $I_{\mathrm{CC}}$ of 1.0 $\mathrm{mA}$ exhibiting the gradual RESET transition for two different RESET stop voltages $\left(V_{\text {RESET-STOP }}=-1.4 \mathrm{~V} \&-2.0 \mathrm{~V}\right)$. The higher RESET voltage toggles the device into a higher $R_{\text {HRS }}$ value [15, 28-30], which subsequently results in a higher SET voltage. This phenomenon is attributed to the fact that under the electric field during RESET, the $\mathrm{V}_{\mathrm{O}}$ 's drift away from the high WF metal electrode resulting in an increase of the effective Schottky barrier height. This drift, however, builds up a concentration gradient giving rise to an opposite diffusion flow of $\mathrm{V}_{\mathrm{O}}$. Hence, the $V_{O}$ distribution approaches an equilibrium (balance) over time [15]. This equilibrium profile is frozen in when the voltage is switched off and depends on the maximum applied RESET voltage. When the RESET voltage increases, the $\mathrm{Ta}_{2} \mathrm{O}_{5}$ close to the high WF metal electrode becomes more depleted from $\mathrm{V}_{\mathrm{O}}$ 's and, thus, the resulting $R_{\mathrm{HRS}}$ value becomes higher [15, 31-34]. The effects of the $V_{\text {RESET-STOP }}$ on the $R_{\text {HRS }}$ for the $\mathrm{W}$ - and the Ta$\mathrm{OE}$ based $\mathrm{Ta}_{2} \mathrm{O}_{5}$ devices are investigated in the range of $-1.4 \mathrm{~V}$ to $-2.0 \mathrm{~V}$ with $-0.2 \mathrm{~V}$ increment under identical SET $I_{\mathrm{CC}}$ of $1.0 \mathrm{~mA}$ using quasi-static voltage sweeps. The $\mathrm{W}-\mathrm{OE}$ devices consistently yield higher $R_{\text {HRS }}$ than the Ta-OE devices, shown in Fig. $4(\mathrm{~b})$. For $V_{\text {RESET-STOP }}=-1.4 \mathrm{~V}$, the $R_{\text {HRS }}$ value of the $\mathrm{Ta}_{2} \mathrm{O}_{5}$ ReRAM with the W-OE is $23 \mathrm{k} \Omega$ in comparison with $2.0 \mathrm{k} \Omega$ for
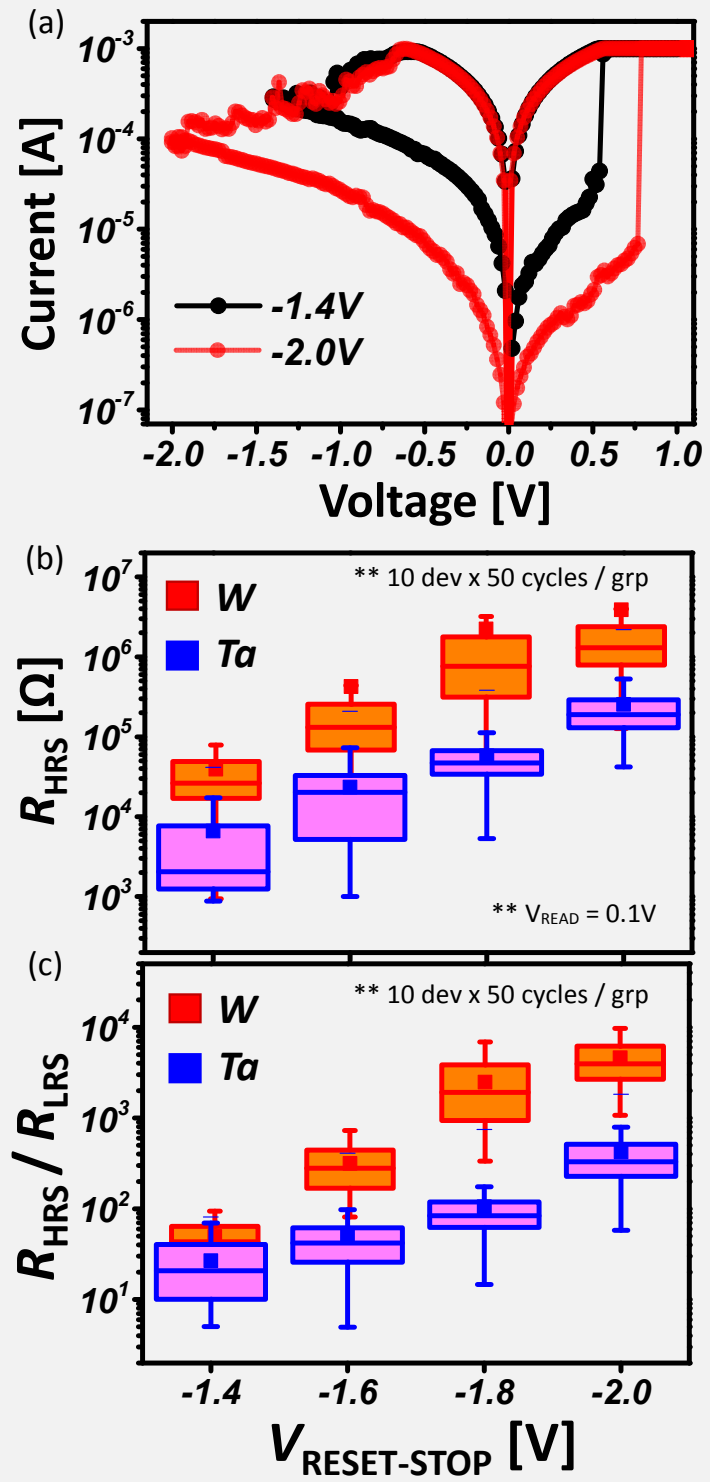

Fig. 4. (a) Typical SET-RESET characteristics of the $\mathrm{Ta}_{2} \mathrm{O}_{5}$ ReRAM device for two different $V_{\text {RESET-STOP }}$ conditions $\left(-1.4 \mathrm{~V},-2.0 \mathrm{~V}\right.$ ), (b) $R_{\text {HRS }}$ change depending on $V_{\text {RESET-STOP }}$ increment for two ohmic electrode materials (W and Ta), (c) Comparison of $R_{\text {HRS }} / R_{\text {LRS }}$ ratio as a function of the $V_{\text {RESET-STOP }}$ increment for the Ta- and the W-OE ReRAM device.

the Ta-OE devices. At $V_{\text {RESET-STOP }}=-2.0 \mathrm{~V}$, the $R_{\text {HRS }}$ value is further increased to $1.2 \mathrm{M} \Omega$ and $200 \mathrm{k} \Omega$ for the $\mathrm{W}$ - and the Ta-OE device, respectively. Due to the high $R_{\mathrm{HRS}}$ for the W-OE device with similar $R_{\mathrm{LRS}}$, the $R_{\mathrm{HRS}} / R_{\mathrm{LRS}}$ ratio of the W-OE based $\mathrm{Ta}_{2} \mathrm{O}_{5}$ device is more than 1,000 at $V_{\text {RESET-STOP }}=-1.8 \mathrm{~V}$. This is about 12 times higher than the one for the Ta-OE device as shown in Fig. 4(c). The AC RESET behavior of the Ta- and W-OE based $\mathrm{Ta}_{2} \mathrm{O}_{5}$ devices is further investigated using voltage pulses of $100 \mathrm{~ns}$ and $100 \mu$ s length. The measurement schematics of AC pulse sequences are shown in Fig. 5(a). The $R_{\text {LRS }}$ of all devices is always kept constant to about $400 \Omega$, which is verified by a $0.1 \mathrm{~V}$ read pulse with $1.0 \mu \mathrm{s}$ width before the RESET pulse application. Fig. 5 (b) shows the $R_{\text {HRS }}$ change for different RESET voltages ranging 
(a)

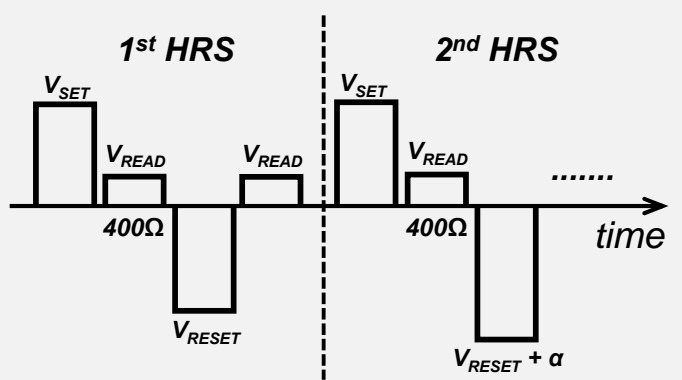

(b)

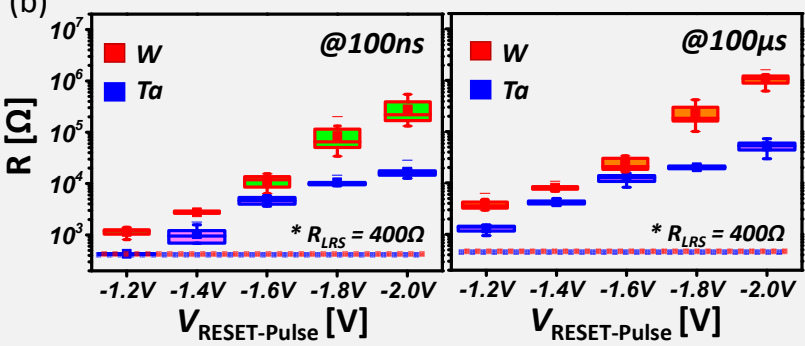

Fig. 5. (a) Schematics of pulse application during the AC measurement, (b) Comparison of the RESET kinetics between the W- and Ta-OE with $100 \mathrm{~ns}$ and $100 \mu$ s pulse width showing resistance change over applied RESET voltage.

from $-1.2 \mathrm{~V}$ to $-2.0 \mathrm{~V}$ at both $100 \mathrm{~ns}$ and $100 \mu \mathrm{s}$ pulse widths. For both, the W- and Ta-OE devices, the $R_{\text {HRS }}$ state varies with the pulse voltage amplitude. For both pulse widths, the rate of resistance change over voltage amplitude is steeper for the $\mathrm{W}$ $\mathrm{OE}$ than that of the Ta-OE device. For the $100 \mathrm{~ns}$ pulse width, at the $V_{\text {RESET-PULSE }}=-2.0 \mathrm{~V}$, the $R_{\text {HRS }}$ of the $\mathrm{W}-\mathrm{OE}$ based $\mathrm{Ta}_{2} \mathrm{O}_{5}$ device was approximately 15 times ( $221 \mathrm{k} \Omega$ for $W$ vs. $16 \mathrm{k} \Omega$ for Ta) higher than that of Ta-OE device. For the longer RESET pulse $(100 \mu \mathrm{s})$ at $V_{\text {RESET-Pulse }}=-2.0 \mathrm{~V}$, the resistance of the $\mathrm{W}$-OE device was about 20 times ( $1.1 \mathrm{M} \Omega$ for $W$ vs. $53 \mathrm{k} \Omega$ for Ta) higher than that of Ta-OE device. As expected, longer pulses $(100 \mu \mathrm{s})$ result in a higher resistance change than the short pulses (100 ns) for both device stacks at given voltage amplitude [35]. The RESET pulse experiment is consistent with the DC-RESET results where the W-OE shows higher $R_{\mathrm{HRS}}$ at any given RESET voltage. The results from both $A C$ and $D C$ measurements thus confirm that the OE material affects the RESET switching speed and equilibrium in the device, which verifies the presumption that oxygen exchange at the $\mathrm{OE} /$ metal-oxide interface actively contributes to the switching process.

Next, the SET kinetics of the $\mathrm{Ta}_{2} \mathrm{O}_{5}$ device for both OE have been investigated with varying amplitudes $V_{\text {SET-Pulse, }}$ as shown in Fig. 6(a). The transient SET pulse (in gray) and its corresponding transient SET current (/SET-Pulse) in blue is measured for $V_{\mathrm{SET} \text { - }}$ Pulse $=1.2 \mathrm{~V}$ and $1.45 \mathrm{~V}$ with Ta-OE device. Prior to the SET pulse application, the $R_{\text {HRS }}$ value for both conditions was kept between $150 \mathrm{k} \Omega$ to $200 \mathrm{k} \Omega$. In the beginning of the $V_{\text {SET-Pulse, the }}$ corresponding $I_{\text {SET-Pulse }}$ is relatively low. However, after some time the current level shows a sudden increase. The point in time at which this abrupt increase in the current is observed, is defined as SET switching time, $t_{\mathrm{SET}}$. For the lower $V_{\mathrm{SET} \text {-Pulse }}$ $(1.2 \mathrm{~V})$, a longer $t_{\mathrm{SET}}(45 \mathrm{~ns})$ is required to (a)
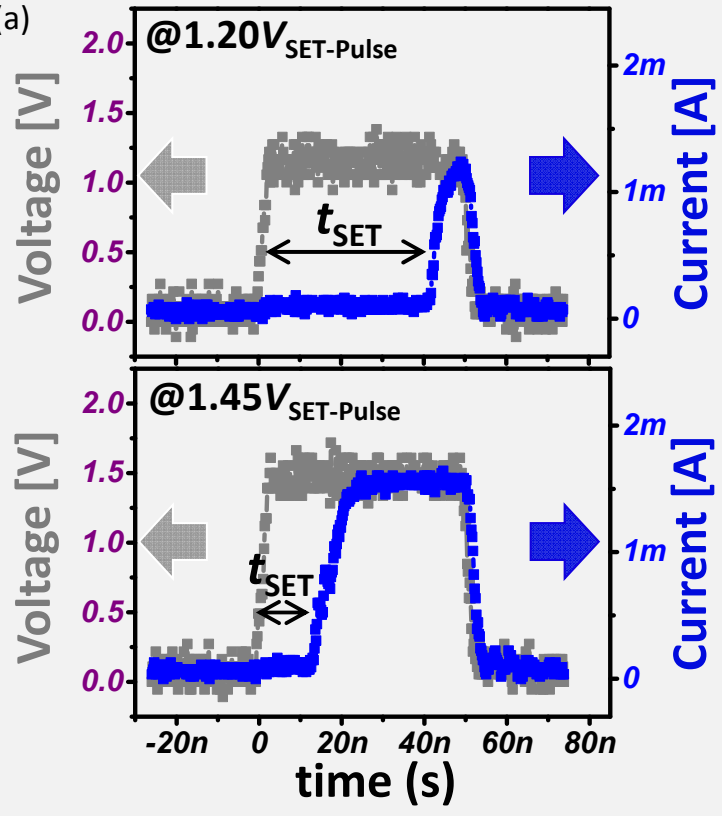

(b)

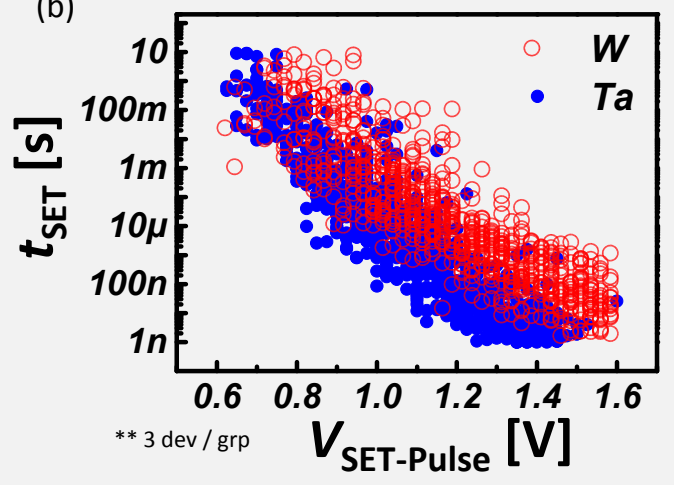

Fig. 6. (a) Examples of the measured transient current (in blue) and the applied SET pulse (in gray) of $V_{\text {SET }}=1.3 \mathrm{~V}$ and $1.75 \mathrm{~V}$. The abrupt increase of current is identified as the SET process. The SET time $\left(t_{\mathrm{SET}}\right)$ is obtained depending on the amplitude of SET pulse. (b) A correlation between the $\mathrm{t}_{\mathrm{SET}}$ and the SET Pulse amplitude for both W- (in red) and Ta-OE (in blue) illustrating the ultra-high nonlinearity of the SET kinetics.

switch the device to the LRS whereas a shorter $t_{\mathrm{SET}}(15 \mathrm{~ns})$ is observed at the higher $V_{\text {SET-Pulse }}(1.45 \mathrm{~V})$. This measurement has been carried out over a wide $V_{\text {SET-Pulse }}$ range spanning from $0.6 \mathrm{~V}$ to $1.6 \mathrm{~V}$ with $25 \mathrm{mV}$ increment for each $\mathrm{OE}$, shown in Fig. 6(b). A clear correlation between $V_{\text {SET-Pulse }}$ and $t_{\text {SET }}$ is observed for both devices. As the $V_{\text {SET-Pulse }}$ increases, the $t_{\text {SET }}$ becomes shorter as reported for other devices [36-38]. For the complete voltage range the Ta-OE devices show the shorter $t_{\mathrm{SET}}$ i.e. faster SET process. So, the transition from $R_{\text {HRS }}$ to $R_{\text {LRS }}$ for the SET process appears faster with $\mathrm{Ta}-\mathrm{OE}$, which is the exactly opposite to the RESET process of the device (that is faster for the $\mathrm{W}-\mathrm{OE}$ ). This outcome further supports the assumption that the OE plays an active role during the switching process. 


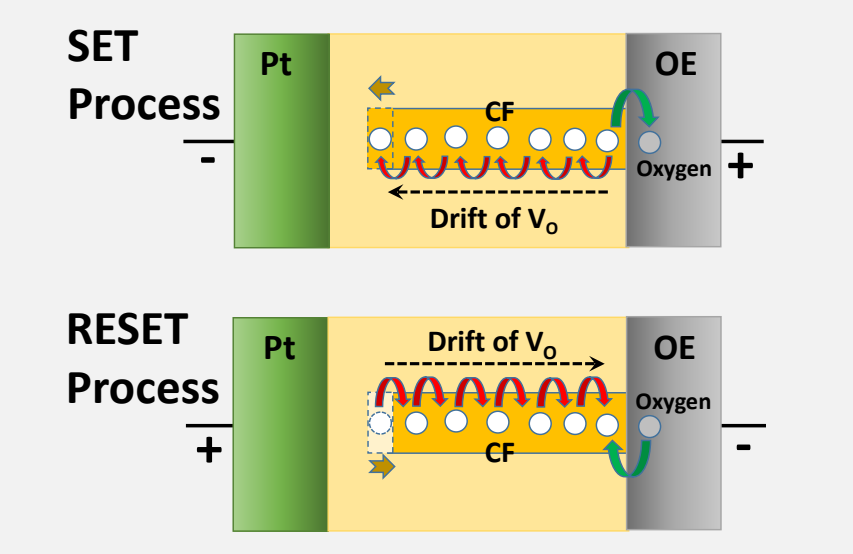

Fig. 7. Illustration showing the movements of oxygen vacancies and oxygen ions during the SET and the RESET process.

\section{Discussion}

The experimental results indicate that the OE plays an active role during the switching process in VCM ReRAM devices. In analogy to the forming process, an oxygen exchange reaction occurs during switching at the OE/metal-oxide interfaces. Thus, the former assumptions of the switching process, in which the amount of $V_{O}$ in the filament stays constant needs to be modified [39]. In addition to the movement of the $V_{0}$ 's in the filament, an oxygen exchange takes place at the OE/oxide interface. Hence, the total amount of the $V_{0}$ 's within the filament changes during the switching process. This modified switching model is illustrated in Fig. 7. During the SET transition, the oxygen vacancies drift towards the high WF metal electrode and decrease the Schottky barrier height. This is corresponding to oxygen moving towards the OE interface, where due to the oxygen exchange reaction oxygen is extracted from the metaloxide and incorporated in the OE. By this, the electrode may be locally oxidized forming a sub-oxide (which would be still be very conductive), while the total amount of the $V_{O}$ in the metaloxide increases (cf. equation 3 , proceeding to the left). During the RESET process, the opposite movement of the $V_{O}$ 's result in their depletion at the high WF metal electrode and accumulation at the $\mathrm{OE}$ electrode. Due to the oxygen exchange reaction at the electrode, oxygen is incorporated in the oxide layer lowering the amount of vacancies at the OE interface. Thus, the total amount of the $\mathrm{V}_{\mathrm{O}}$ 's decreases in the oxide layer. The rate of this oxygen exchange reaction depends on $E_{\mathrm{vo}}$. $\mathrm{A}$ faster extraction of the oxygen ion from the switching oxide layer (SET) is achieved for a lower (positive) $E_{\mathrm{V} o}$ values. In contrast, the re-incorporation of oxygen ions into the switching oxide layer (RESET) is faster for higher (positive) $E_{\mathrm{vo}}$. Thus, a higher $E_{\mathrm{VO}}$ is beneficial for faster RESET operation.

The experimental observations can now be explained in the context of the modified model. The early RESET failure occurs in the $\mathrm{Ti}$ - and $\mathrm{Hf}-\mathrm{OE}$ devices. For those two electrodes, $E_{\mathrm{VO}}$ is (a)

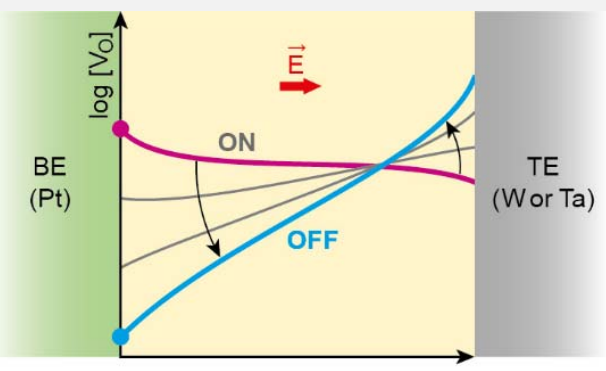

(b)

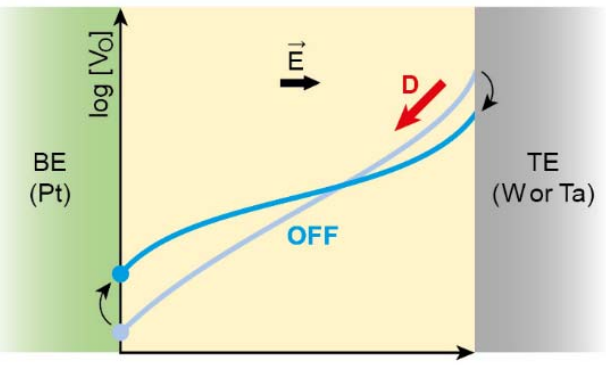

(c)

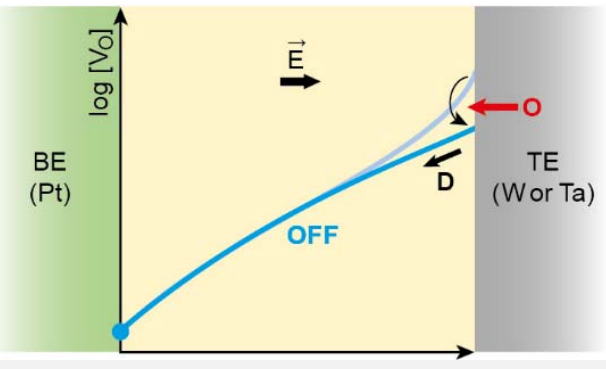

Fig. 8. (a) Redistribution of oxygen vacancies under the electric field $(E)$ during the RESET process. The sketches are based on simulations of $V_{0}$ concentration profiles during ReRAM switching (b) Diffusion of oxygen vacancies counteracting the concentration gradient by the drift process. (c) Oxygen exchange with the Ohmic electrode resulting in lower concentration of oxygen vacancy at the Pt interface.

negative, i.e. there is a continuous thermodynamic drive for $V_{O}$ formation. Kinetic barriers to this may be lowered due to Joule heating during the switching process, with oxygen extraction from $\mathrm{Ta}_{2} \mathrm{O}_{5}$ strongly enhanced during the SET process while $\mathrm{O}$ re-incorporation during RESET process is strongly reduced. Hence, the total amount of the oxygen vacancies is increasing from cycle to cycle, reducing the film resistivity. Eventually, the current and the dissipated power during the RESET process is so high that oxygen may get excorporated at the opposite $\mathrm{Pt}$ electrode (i.e., we get a spurious SET at this Pt electrode), resulting in an overall RESET failure. For the Ta- and W-OE devices, the oxygen exchange during the SET and the RESET process is better balanced and thus stable switching is obtained. The different speed of the RESET process for these devices can also be explained with the oxygen exchange model. Fig. 8 sketches the change in the oxygen vacancy profiles during the RESET process, where the profiles are motivated by previous simulation results $[14,15]$. During the RESET process, the 
oxygen vacancies are depleted near the high WF Pt electrode and pile up at the $O E$ interface by redistribution of charged oxygen vacancies ( $V o ̈)$ under the electric field $(E)$, as shown in Fig. 8(a). The depletion at the high WF Pt electrode results in a space charge region and a high barrier height blocking the electron conduction. The diffusion (D) of the $V_{O}$ will counteract the concentration gradient build-up by the drift, resulting in equilibrium in Fig. $8(\mathrm{~b})$. By that, the $\mathrm{V}_{\mathrm{O}}$ accumulation at the $\mathrm{OE}$ will be reduced. However, these amount of the $V_{O}$ will diffuse towards the high WF Pt electrode, decreasing the depletion and increasing the current in the $R_{\mathrm{HRS}}$. The oxygen (O) exchange with the $\mathrm{OE}$ will reduce the high $\mathrm{V}_{\mathrm{O}}$ concentration lowering the backdiffusion force (D), shown in Fig. 8(c). As the result, a lowered concentration of the $V_{O}$ at the high WF Pt interface can be maintained and the leakage current remains low. The W-OE can release oxygen (O) back easier than the Ta-OE due to its higher energy for defect formation. This is why the W-OE can achieve lower leakage current (higher $R_{\text {HRS }}$ ) with the same $V_{\text {RESET }}$ application. In contrast, for the switching speed of the SET process, the Ta-OE is faster than the W-OE. This is attributed to the speed of the oxygen exchange reaction at the interface. The extraction of the oxygen ions at the $\mathrm{OE}$ electrode is faster for the Ta-OE because it has the lower $E_{\mathrm{vo}}$.

\section{Conclusions}

In this study, $\mathrm{Ta}_{2} \mathrm{O}_{5}$-based ReRAM devices have been studied with $\mathrm{W}, \mathrm{Ta}, \mathrm{Ti}$ and $\mathrm{Hf}-\mathrm{OEs}$. Based on our experimental observations, a modified switching model is proposed. In contrast to the previous models, the experimental results show that oxygen exchange reactions with the ohmic electrode not only occur during deposition and the electroforming process, but also during each SET and RESET process. The relevant design property presumably is the interfacial oxygen defect formation energy $E_{\mathrm{V}}$ at the $\mathrm{OE} /$ oxide interface. The Ti- and $\mathrm{Hf}-\mathrm{OE}$ devices suffer from an early RESET failure due to an accumulation of the $\mathrm{V}_{\mathrm{O}}$ 's during the switching cycles. This is explained by their negative $E_{\mathrm{vo}}$, which favors the reduction of the switching oxide layer. In contrast, the positive $E_{\mathrm{VO}}$ for the Ta- and W-OE leads to highly stable switching process in the $\mathrm{Ta}_{2} \mathrm{O}_{5}$ ReRAM. The W-OE devices show an increased $R_{\text {HRS }}$ compared with the Ta-electrode under identical RESET conditions. This difference of the $R_{\text {HRS }}$ value can also be explained by the oxygen exchange reaction. The $E_{\mathrm{vo}}$ of $\mathrm{W} / \mathrm{Ta}_{2} \mathrm{O}_{5}$ favors incorporation of the oxygen ions into the switching oxide layer decreasing the total amount of the $V_{O}$ inside the switching oxide, which will lower the current in high resistance state under the given biasing conditions of the RESET process. On the opposite side, the lower $E_{\mathrm{VO}}$ for Ta-OE results in faster SET processes than its counterpart W-OE. These observations indicate that oxygen interchange with the OE plays an important role during the resistive switching, leading to extended modified switching model. The proposed model can explain the experimental dependence of the resistive switching properties as RESET depth and SET speed on the used material stack, and so will be beneficial for the future material's design of optimized ReRAM devices.

\section{Acknowledgements}

This work was supported by the German Research Foundation (DFG) within the framework of SFB 917, Nanoswitches and the authors thank Dr. Daesung Park and Mr. Sebastian Zischke from Central Facility for Electron Microscopy (GFE), RWTH Aachen for the cross-sectional TEM images, and Dr.llia Valov from Peter Grünberg Institute, Forschungszentrum Jülich for critical review of the manuscript.

\section{References}

1. M.-J. Lee, C. B. Lee, D. Lee, S. R. Lee, M. Chang, J. H. Hur, Y.-B. Kim, C. -J. Kim, D. H. Seo, S. Seo, U.-I. Chung, I.-K. Yoo, and K. Kim, Nature Materials, 2011, 10, 625.

2. M. Fujimoto, H. Koyama, M. Konagai, Y. Hosoi, K. Ishihara, S. Ohnishi, and N. Awaya, Applied Physics Letters, 2006, 89, 223509/1.

3. R. Waser and M. Aono, Nature Materials, 2007, 6, 833.

4. A. Wedig, M. Luebben, D.-Y. Cho, M. Moors, K. Skaja, V. Rana, T. Hasegawa, K. Adepalli, B. Yildiz, R. Waser, and I. Valov, Nature Nanotechnology, 2016, 11, 67.

5. F. A. Kroeger and H. J. Vink, Solid State Physics, 1956, 3, 307.

6. D. lelmini and R. Waser, Wiley-VCH, 2016

7. S. Stille, C. Lenser, R. Dittmann, A. Koehl, I. Krug, R. Muenstermann, J. Perlich, C. M. Schneider, U. Klemradt, and R. Waser, Applied Physics Letters, 2012, 100, $223503 / 1$

8. A. Sharma, M. Noman, M. Abdelmoula, M. Skowronski, and J. Bain, Advanced Functional Materials, 2014, 24, 5522.

9. R. Waser and M. Wuttig, 2009 Proceedings of Esscirc, 2009, 66.

10. J. J. Yang, F. Miao, M. D. Pickett, D. A. A. Ohlberg, D.R. Stewart, C. N. Lau, and R. S. Williams, Nanotechnology, 2009, 20, 215201

11. R. Waser, R. Dittmann, G. Staikov, and K. Szot, Advanced Materials, 2009, 21, 2632.

12. J. J. Yang, M. D. Pickett, X. Li, D. A. A. Ohlberg, D. R Stewart, and R. S. Williams, Nature Nanotechnology, 2008, 3, 429.

13. J. H. Hur, M.-J. Lee, C. B. Lee, Y.-B. Kim, and C.-J. Kim, Phys. Rev. B, 2010, 82, 155321 
14. A. Marchewka, R. Waser, and S. Menzel, 2015 International Conference On Simulation of Semiconductor Processes and Devices (SISPAD), 9-11 September, Washington D.C, USA, 2015, 297.

15. A. Marchewka, B. Roesgen, K. Skaja, H. Du, C.-L. Jia, J. Mayer, V. Rana, R. Waser, and S. Menzel, Advanced Electronic Materials, 2016, 2

16. A. C. Torrezan, J. P. Strachan, G. Medeiros-Ribeiro, and R. S. Williams, Nanotechnology, 2011, 22, 485203.

17. C. Chen, L. Goux, A. Fantini, A. Redolfi, S. Clima, R. Degraeve, Y. Chen, G. Groeseneken, and M. Jurczak, Technical Digest - International Electron Devices Meeting, IEDM, 2015, 2015-February, 14.2.1.

18. L. Goux, A. Fantini, Y. Y. Chen, A. Redolfi, R. Degraeve, and M. Jurczak, Ecs Solid State Letters, 2014, 3, Q79.

19. C. Hsu, C. Wan, I. Wang, M. Chen, C. Lo, Y. Lee, W. Jang, C. Lin, and T. Hou, Electron Devices Meeting (IEDM), 2013 IEEE International, 2013, 10.4.1.

20. A. Prakash, D. Jana, and S. Maikap, Nanoscale Research Letters, 2013, 8, 1.

21. W. Kim, B. Rösgen, T. Breuer, S. Menzel, D. Wouters, R. Waser, and V. Rana, Microelectronic Engineering, 2016, 154, 38.

22. J. J. Yang, J. P. Strachan, F. Miao, M. Zhang, M. D. Pickett, W. Yi, D. A. A. Ohlberg, G. Medeiros-Ribeiro, and R. S. Williams, Applied Physics A: Materials Science \& Processing, 2011, 102, 785.

23. Y. S. Chen, H. Y. Lee, P. S. Chen, P. Y. Gu, W. H. Liu, W. S. Chen, Y. Y. Hsu, C. H. Tsai, F. Chen, M. J. Tsai, and C. Lien, IEEE Electron Device Letters, 2011, 32, 390.

24. Y. Guo and J. Robertson, Applied Physics Letters, 2014, 105, 222110.

25. Y. Guo and J. Robertson, Applied Physics Letters, 2014, 105, 223516/1.

26. J. Robertson and S.J. Clark, Physical Review B: Condensed Matter, 2011, 83, 0752051.

27. Y. Guo and J. Robertson, Applied Physics Letters, 2014, 105, 223516.

28. L. Goux, Y. Chen, L. Pantisano, X. Wang, G. Groeseneken, M. Jurczak, and D. J. Wouters, Electrochemical and Solid State Letters, 2010, 13, G54.
29. F. Nardi, S. Larentis, S. Balatti, D. Gilmer, and D. lelmini, IEEE Transactions on Electron Devices, 2012, 59, 2461.

30. S. Yu, Y. Wu, R. Jeyasingh, D. Kuzum, and H. P. Wong, IEEE Transactions on Electron Devices, 2011, 58, 2729.

31. S. Larentis, C. Cagli, F. Nardi, and D. Ielmini, Microelectronic Engineering, 2011, 88, 1119.

32. D. Ielmini, IEEE Transactions on Electron Devices, 2011, 58, 4309.

33. K. Kim, D. S. Jeong, and C. S. Hwang, Nanotechnology, 2011, 22

34. J. Y. Son and Y.-H. Shin, Applied Physics Letters, 2008, 92, 222106.

35. H. Schroeder, V. V. Zhirnov, R. K. Cavin, and R. Waser, Journal of Applied Physics, 2010, 107, 054517/1.

36. S. Menzel, M. Salinga, U. Böttger, and M. Wimmer, Adv. Funct. Mater., 2015, 25, 6306.

37. Y. Nishi, K. Fleck, U. Boettger, R. Waser, and S. Menzel, IEEE Transactions on Electron Devices, 2015, 62, 1561.

38. Y. Nishi, S. Menzel, K. Fleck, U. Boettger, and R. Waser, IEEE Electron Device Letters, 2013, PP, 1.

39. R. Degraeve, A. Fantini, S. Clima, B. Govoreanu, L. Goux, Y.Y. Chen, D.J. Wouters, Ph. Roussel, G.S. Kar, G. Pourtois, S. Cosemans, J.A. Kittl, G. Groeseneken, M. Jurczak, and L. Altimime, Proceedings of the 2012 Symposium on VLSI Technology, 2012, 75. 